\title{
Ultrafast solid-state laser oscillators: a success story for the last 20 years with no end in sight
}

\author{
U. Keller
}

Received: 21 April 2010 / Published online: 13 May 2010

(c) The Author(s) 2010. This article is published with open access at Springerlink.com

\begin{abstract}
Ultrashort lasers provide an important tool to probe the dynamics of physical systems at very short timescales, allowing for improved understanding of the performance of many devices and phenomena used in science, technology, and medicine. In addition ultrashort pulses also provide a high peak intensity and a broad optical spectrum, which opens even more applications such as material processing, nonlinear optics, attosecond science, and metrology. There has been a long-standing, ongoing effort in the field to reduce the pulse duration and increase the power of these lasers to continue to empower existing and new applications. After 1990, new techniques such as semiconductor saturable absorber mirrors (SESAMs) and Kerrlens mode locking (KLM) allowed for the generation of stable pulse trains from diode-pumped solid-state lasers for the first time, and enabled the performance of such lasers to improve by several orders of magnitude with regards to pulse duration, pulse energy and pulse repetition rates. This invited review article gives a broad overview and includes some personal accounts of the key events during the last 20 years, which made ultrafast solid-state lasers a success story. Ultrafast Ti:sapphire, diode-pumped solid-state, and novel semiconductor laser oscillators will be reviewed. The perspective for the near future indicates continued significant progress in the field.
\end{abstract}

\section{Current status and introduction}

As we look back from today in 2010 for the last 20 years, we see that ultrafast solid-state lasers have become the key

\section{U. Keller $(\varangle)$}

Institute of Quantum Electronics, Physics Department, ETH

Zurich, Zurich, Switzerland

e-mail: keller@phys.ethz.ch enabling technology for many new applications, and have established themselves successfully in at least several industrial areas. The situation was substantially different 20 years ago, when ultrafast lasers were predominantly based on dye laser technology or active modelocked solid-state lasers, and it was assumed that passive modelocking of diode-pumped solid-state lasers was very difficult, if not impossible. Key breakthroughs came with the invention of the semiconductor saturable absorber mirror (SESAM) [1, 2] and Kerr-lens modelocking (KLM) [3]. Previously, Q-switching instabilities prevented stable passive modelocking of diode-pumped solid-state lasers for more than 25 years. This breakthrough was enabled through the major progress in solid-state laser technology: first with the discovery of the Ti:sapphire laser material [4] and second with the rapid progress in highpower semiconductor diode-laser arrays to efficiently pump solid-state lasers.

For this invited review paper for the special celebration of Volume 100 in Applied Physics B, I will provide some more details describing the events that led to the rapid progress in ultrafast solid-state lasers. I have been actively involved at the frontier of this field for more than 20 years and can provide some more personal insight into how the field evolved. Furthermore, many important results have been published in Applied Physics B over this period. I will start with a brief description of the status 20 years ago, then continue with the invention of KLM, the SESAM, and the frequency comb technique. Towards the end I will give a brief summary of the many different applications that have been enabled by this rapid progress in ultrafast solid-state lasers (such as material processing, frequency metrology, attosecond science and many others) and finally conclude with an outlook into the current research efforts and challenges. I will restrict this review to laser oscillators and will not review the fast and important progress achieved with ultrafast amplifier systems to date. 
Over the years I have been invited to write many papers and book chapters on this subject where more technical and scientific details are provided. For the interested readers I recommend my recent extensive review from 2007 on ultrafast solid-state lasers in the Landolt-Börnstein Series [5] which also provides detailed tables listing the different results obtained with passively modelocked solid-state laser oscillators. This is an updated and expanded review of a previous invited publication in Progress in Optics in 2004 [6]. Many results from different groups have been listed and discussed which cannot be repeated in this shorter review paper, and I apologize in advance that I cannot give full credit to so many contributions, or in the case that I have inadvertently omitted any contributions. For a quick overview of the many interesting applications, I recommend the short invited review in Nature from 2003 [7]. For the ultrafast semiconductor lasers based on the SESAM-VECSEL approach I recommend a recent invited paper authored by myself und Anne Tropper in 2006 [8] which again includes many useful tables listing results achieved at that time - an ideal starting point for researchers who want to get started in this new field. So far research and progress in ultrafast solidstate lasers have kept going strong and many of the new results are not included in these recent reviews. I therefore briefly describe the current performance frontier in the next few paragraphs. In contrast to the mentioned review papers, I will present in the following sections a more personal report which describes in more detail how certain key events happened.

\subsection{Current frontier in KLM Ti:sapphire laser oscillators}

Today KLM Ti:sapphire lasers can generate the shortest pulses ever produced directly from a laser oscillator, with pulse durations in a regime where the full-width halfmaximum (FWHM) pulse duration contains only one to two optical cycles. One optical cycle at $800 \mathrm{~nm}$ is only $2.7 \mathrm{fs}$ long. The long-standing record of 6-fs pulses [9] in the dye laser era in 1987 was passed for the first time in 1999, more than 10 years later, with a KLM SESAM-assisted Ti:sapphire laser using double-chirped mirrors (DCMs) for broadband dispersion compensation [10]. In contrast to the older world-record result no external pulse amplification and compression was necessary and these pulses were obtained directly at the output of the laser oscillator. This new world record was reproduced and confirmed very soon afterwards [11, 12] (see the addenda [12] for the changes made during the reviewing process) and further improved towards the 5-fs regime with better DCMs [13]. The DCMs [14] were based on the initial invention of the chirped mirrors [15] but offered a more analytical approach for the chirped mirror design and explained the residual group delay dispersion (GDD) oscillations typically observed from such mirrors [16].

\subsection{Current frontier in SESAM-modelocked solid-state} laser oscillators

Aside from ultrashort or broadly tunable Ti:sapphire lasers based on KLM, most other solid-state lasers currently use passive modelocking with the SESAM approach. Today SESAM-modelocked solid-state laser oscillators have pushed the pulse energy by four orders of magnitude from the $1-n J$ to the $10-\mu \mathrm{J}$ level without any further amplification [17]. The current frontier is at pulse energies of up to $11.3 \mu \mathrm{J}$ with the thin-disk gain element in a single folding mirror configuration [18] and up to $25.9 \mu \mathrm{J}$ in an active multi-pass cavity [19] with 700 to 800 fs pulses at a few $\mathrm{MHz}$ pulse repetition rates using Yb:YAG thin-disk lasers. Furthermore, the average output power has been pushed beyond the 100-W level for the first time with a SESAMmodelocked $\mathrm{Yb}: \mathrm{Lu}_{2} \mathrm{O}_{3}$ thin-disk laser oscillator generating $141 \mathrm{~W}$, with 738 fs pulses, at $60 \mathrm{MHz}$ repetition rate, with a pulse energy of $2.4 \mu \mathrm{J}$ with an optical-to-optical efficiency of more than $40 \%$ [20].

SESAM modelocking also has pushed the frontier in gigahertz pulse repetition rates from diode-pumped solid-state lasers by two orders of magnitude, to $160 \mathrm{GHz}$ at $1 \mu \mathrm{m}$ [21] and $100 \mathrm{GHz}$ at $1.5 \mu \mathrm{m}[22,23]$. These lasers demonstrate superior noise performance and are close to being quantum noise limited [24], which are much better than fiber or edge-emitting semiconductor lasers because of their high-Q (low-loss) cavities. Many applications benefit from such low-noise performance: for example with a SESAMmodelocked Er:Yb:glass laser excellent frequency comb stability was demonstrated with a very high signal-to-noise level for the self-referenced carrier-envelope offset (CEO) frequency noise beat [25] with rather long pulses and without any further amplification before the broadband continuum generation [26].

\subsection{Current Frontier in Optically-Pumped VECSEL and MIXSELS}

The work on SESAM-modelocked solid-state lasers motivated novel ultrafast semiconductor lasers and allowed for the first demonstration of a passively modelocked optically-pumped vertical external cavity surface-emitting laser (VECSEL) in 2000 [27]. Since then we have observed rapid progress with average power scaling to more than $2 \mathrm{~W}$ with transform-limited picosecond pulse duration [28] and by Anne Tropper's group with shorter pulse durations into the sub-100-femtosecond regime [29]. In addition a new concept was invented, since both the gain and the absorber are based on semiconductor materials and therefore can be integrated within one wafer, which resulted in the first demonstration of a MIXSEL (Modelocked Integrated eXternal-cavity Surface-emitting Laser) [30, 31]. 
With progress in quantum-dot saturable absorbers [32] an optically-pumped antiresonant MIXSEL design [33] became possible, which allowed for average power scaling up to the unprecedented level of more than $6 \mathrm{~W}$ to date [34]. We are currently exploring the possibilities to extend these concepts towards electrical pumping [35] and femtosecond pulse generation. The compactness and the simplicity of the MIXSEL platform appears well-suited for cost-efficient mass production [31] and has the potential to provide ultrafast lasers for applications where the current ultrafast laser technology is still considered to be too expensiveeven though we made tremendous progress in this regard with SESAM-modelocked diode-pumped solid-state lasers, which ultimately enabled modelocked lasers in industrial mass production.

\subsection{Current frontier in SESAM Q-switched microchip lasers}

SESAM Q-switched microchip lasers bridge the gap between modelocking and Q-switching in terms of pulse duration and pulse repetition rates. My group pioneered this work and we achieved cutting-edge results and developed simple design guidelines to optimize pulse energy, pulse duration and pulse repetition rate for different application specifications [36].

We became involved with Q-switched microchip lasers because our early attempts to passively modelock multigigahertz diode-pumped solid-state lasers failed, due to Qswitching instabilities (see Sect. 1.3). Even though no modelocking was observed initially, we obtained however stable passive Q-switching from a Nd:LSB microchip laser with an attached SESAM for the first time in 1996 [37]. We ultimately stopped our research efforts for these lasers many years ago because industrial applications were hindered by some microchip laser patents.

Pulses as short as $37 \mathrm{ps}$ have been generated with a SESAM Q-switched Nd:Vanadate microchip laser, which are the shortest pulses produced from a Q-switched solidstate lasers [36]. The pulse repetition rate can be controlled by the pump power because it is proportional to the small signal gain in the microchip laser, and a typical repetition rate ranging between a few $100 \mathrm{~Hz}$ to $10 \mathrm{MHz}$ could be demonstrated. The highest pulse energies from SESAM Qswitching were demonstrated with a Yb:YAG microchip laser generating a $1.1-\mu \mathrm{J}$ pulse energy with $530 \mathrm{ps}$ pulse durations at $12 \mathrm{kHz}$ pulse repetition rate [38] and with an Er:Yb:glass microchip laser generating $11.2 \mu \mathrm{J}, 840 \mathrm{ps}$, at $1.4 \mathrm{kHz}$ [39] in the eye-safe wavelength regime of $1.5 \mu \mathrm{m}$. Most recently it seems that the community interest is picking up and we could observe different groups reporting on new results on SESAM Q-switched microchip lasers during the last Advanced Solid-State Photonics Topical meeting of the OSA in Feb. 2010.

\section{Status 20 years ago}

It is worthwhile to remember that the current status and understanding, which we usually take for granted today, was often either assumed not to be possible and/or we had no idea how to solve long-term ongoing problems, when looking back twenty years ago to around 1990. Relevant and important examples are:

- diode-pumped solid-state lasers could not be passively modelocked without Q-switching instabilities-theories even further established the commonly accepted knowledge that this does not work

- novel concepts based on coupled-cavity modelocking were assumed to finally resolve these problems but added a lot of other problematic issues

- novel Ti:sapphire lasers generated pulses without any known explanation or any visible saturable absorber inside and were in contradiction to the established modelocking theories

- stable passive modelocking was obtained with long open net gain windows after the pulse-not clear why noise would not grow in these windows and ultimately destabilize the pulse generation process

All these problems ultimately resulted in an explosion of activities, which made modelocking of solid-state lasers a hot topic for the last 20 years. In the following I would like to explain these examples in more details.

\subsection{Q-switching instabilities}

Early attempts to passively modelocked solid-state lasers used intracavity dye saturable absorbers. All these attempts resulted in Q-switching instabilities which prevented a stable pulse train with constant pulse parameters. In the best case stable Q-switched modelocking was achieved where the pulse train was intensity modulated by Q-switched macropulses at the pulse repetition rate close to the relaxation oscillations of the solid-state laser. This was also the case in the first dye saturable absorber modelocked solidstate laser by DeMaria and co-workers in 1966 [40].

All these unsuccessful attempts ultimately were summarized and explained by a theory paper by Haus in 1976 [41] where he investigated the parameter regime of saturable absorbers and explained why it is practically impossible to obtain passive modelocking from solid-state lasers. I was made aware of this paper during my Ph.D. thesis work in Stanford (1985-1989) during which my Ph.D. advisor Prof. Dave Bloom sent me to Prof. Anthony Siegman, one of the pioneers in active modelocking theories [42, 43], to find out why we cannot passively modelock Nd:glass lasers. At that time we used 30 to 100 ps pulses from flashlamppumped Nd:YAG and Nd:YLF lasers which we had to externally compress into the few picosecond regime [44] for 
electro-optic sampling applications [45]. We needed even shorter pulses, which we achieved with a double-stage pulse compressor-rather tricky to use, to say the least. At that time the only alternatives were dye lasers $[46,47]$ or colorcenter lasers $[48,49]$ which were costly, difficult to operate, and needed extensive maintenance, and therefore not very attractive even to a university laboratory with graduate students. Hence Bloom sent me to the local expert to explore the possibilities to passively modelock Nd:glass lasers, motivated by the fact that these lasers have a much broader gain bandwidth than the Nd-doped YAG or YLF laser. Siegman at that time pulled out the mentioned Haus paper [41] to inform me that this does not work and this fact had been established a long time ago. This basically stopped any efforts along those lines in the Bloom group. Instead I continued to concentrate my efforts on improved active modelocking of diode-pumped solid-state lasers, which ultimately resulted in the first sub-10-ps pulses directly generated from a Nd:YLF laser oscillator [50, 51].

During the 1980s we observed tremendous progress in diode-pumped solid-state lasers, with rapid improvements in high-brightness pump diodes replacing expensive, powerhungry flashlamps. Restricting the pulse generation method to active modelocking was a challenge blocking further simplification and cost reduction. A passively modelocked laser provided an attractive step forward compared to active modelocking, since it consists in essence of the laser gain element, the loss or modulator element, and the mirrors (output coupler and high reflector) forming the laser cavity. Passive modelocking distinguishes itself from active modelocking in that there is no "active" drive signal required to form the optical pulses (i.e. electrically-driven optical modulator). The optical pulse itself forms "passively" the required loss modulation. The benefit of this approach is its simplicity, robustness, and generally superior performance compared to active modelocking.

The Q-switching problem for passively modelocked diode-pumped solid-state lasers was ultimately solved with SESAMs (see more in Sect. 4). In contrast to dye saturable absorbers, the SESAMs opened up a completely different regime of saturable absorber parameters that were not considered in the Haus paper of 1976 [41]. In 1999 we resolved this theoretical issue with correct stability criteria against Qswitching instabilities, which were very simple and directly related to the experimental parameters of the laser and the SESAM [52]. We then extended these criteria for inverse saturable absorption that can take place due to two photon absorption (TPA) and defect saturation effects [53].

\subsection{Coupled-cavity modelocking}

The soliton laser [54] was one of the early examples of coupled-cavity modelocking where a color-center laser experienced soliton pulse compression from a coupled cavity with a fiber providing negative GDD, which was responsible for pulse formation. This work led to the accidental discovery of additive pulse modelocking (APM) because the "wrong" fiber with positive instead of negative GDD was used inside a coupled cavity but stable modelocking was still observed in 1989 [55]. This was initially a puzzling result because generally a pulse becomes broadened after propagating in a fiber with positive dispersion. Therefore it was assumed that the nonlinear cavity does not add a pulse shortening mechanism in analogy to the soliton laser where soliton pulse compression is helping in pulse formation. This result was later explained by the so-called APM process [56] where a simple explanation for the pulse formation was given: the pulse in the nonlinear cavity indeed becomes broadened, but self-phase modulation (SPM) still introduces an intensity dependent phase shift, such that the main pulse in the main cavity and the re-injected pulse from the coupled cavity interfere constructively at the peak and destructively at the wings of the pulse, which results in a pulse shortening effect. This however requires interferometric coupled-cavity length control for stable modelocking. APM was very successfully transferred to many different diode-pumped solidstate lasers (see Table 2 in Ref. [5]).

Previously in 1988 numerical simulations showed that any nonlinear coupled cavity can produce pulse shortening [57] but the simple explanation as demonstrated with APM was of course missing. However this paper also motivated my work at Bell Labs in 1990. Instead of a fiber in a coupled cavity I used a semiconductor saturable absorber which I borrowed from my office neighbor (Keith Goossen) who used it for all-optical switching applications. It was a conventional GaAs/GaAlAs quantum-well structure with $75 \mathrm{GaAs}$ quantum wells, which was antireflection coated and was grown on top of a AlAs/GaAlAs dielectric Bragg mirror centered near $850 \mathrm{~nm}$. I placed this absorber mirror inside a coupled cavity of a commercial continuous-wave (cw) Ti:sapphire laser and soon afterwards achieved stable modelocking with picosecond pulses. No interferometric coupled-cavity length control was necessary as before with APM and stable pulse formation was even achieved at larger cavity length detuning in the range of $1 \mathrm{~mm}$ but with longer pulses. This technique was quickly transferred to a diodepumped Nd:YLF laser with similar observations [58]. These results ultimately showed that in contrast to APM no nonlinear phase shift but instead a nonlinear amplitude modulation was used. We then referred to this modelocking technique as resonant passive modelocking (RPM) because a resonant nonlinearity was used $[59,60]$.

RPM ultimately led to the invention of the SESAM in 1992 [1] (see Sect. 4) and the explanation of "magic" modelocking in 1991 [61] (see next section and Sect. 3). Today all these more complicated coupled-cavity modelocking techniques are not used anymore and have been replaced by either SESAM modelocking or KLM. 


\section{3 "Magic" modelocking}

By the 1980s, modelocking theories were well established by the leading work of Siegman [42, 43], New [62], and Haus [63-65]. No additional efforts were considered to be necessary. However the introduction of the new Ti:sapphire laser material [4] changed this drastically. Once these crystals became commercially available, new modelocking results were observed $[3,66]$ and presented at international conferences in 1990 [67, 68]. These results could not be explained by the established modelocking theories.

The first result was built in analogy to the colliding pulse modelocked (CPM) dye laser [69] replacing the dye gain element by the novel Ti:sapphire laser crystal and generated pulses as short as $50 \mathrm{fs}$. However, based on the understanding at that time, this laser should not have worked, since the Ti:sapphire laser did not have the dynamic gain saturation to critically sustain the pulse formation as in a CPM dye laser [70] (Fig. 1a). Instead, the Ti:sapphire laser exhibits a nearly constant gain saturation typically observed for solidstate lasers with small gain cross sections (e.g. more than 1000 times smaller than dye lasers). Thus, for an expert it was clear that the slow dye saturable absorber, with a recovery time in the nanosecond regime, could not support sub-100-fs pulses with a Ti:sapphire laser as before with dye lasers [71].

The second result was presented as a postdeadline paper at CLEO [68] and reported on a surprising result of a Ti:sapphire laser that apparently had no real saturable absorber inside the laser but generated pulses as short as 60 fs. This second result-without any clearly visible saturable absorber-had a major impact in the research community, was initially termed "magic modelocking" and started a major research effort in passive modelocking of solid-state lasers. Sibbett used a coupled-cavity modelocking model, since they had to slightly misalign the cavity for stable modelocking. His explanation assumed a coupledcavity modelocking mechanism based on the nonlinear coupling between the fundamental and higher order transverse modes [3].

Both lasers were not understood, and in the end their operation was accepted to be based on KLM [61, 71] which will be discussed in more details in Sect. 3 .

\subsection{Modelocking theories and long net gain windows}

More than twenty years ago, modelocking theories were well established for active modelocking [42, 43, 63]. In addition, passively modelocked dye lasers were fully explained by a slow saturable absorber modelocking model for which the dynamic gain saturation required a critical balance between gain and absorber saturation to open a net gain window that supports ultrashort pulses (Fig. 1a) [62]. It was always assumed that before and after the pulse the net gain

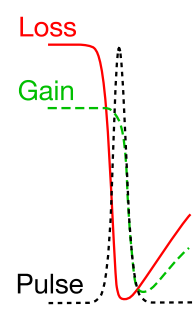

a $\quad$ Time $\rightarrow$

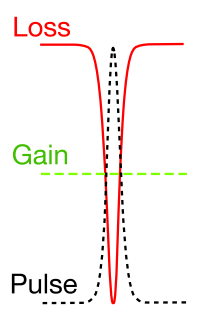

b Time $\rightarrow$

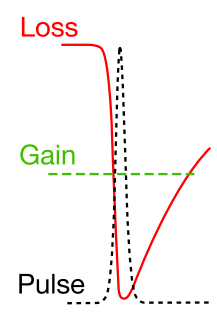

c Time $\rightarrow$

Fig. 1 Summary of the different modelocking techniques: (a) passive modelocking with a slow saturable absorber and dynamic gain saturation [62, 64], (b) passive modelocking with a fast saturable absorber [65] and (c) passive modelocking with a slow saturable absorber without dynamic gain saturation in the picosecond regime [73] and in the femtosecond regime (referred to as soliton modelocking) [74-76]

window needs to be closed for stable modelocking. Therefore, soon after the discovery of KLM it was assumed that this modelocking process is based on a fast saturable absorber model (Fig. 1b) with a fast loss modulation inversely proportional to the pulse intensity [72]. Therefore, even with constant gain saturation the net gain window is only open during the pulse duration.

Soon after the first demonstration of SESAM-modelocked solid-state lasers, it became apparent that much shorter pulses are typically generated with slow saturable absorbers for which the recovery time is typically around 10 to 30 times longer than the pulse duration. This means that the net gain window was open after the pulse (Fig. 1c) and it was initially not clear how this would not destabilize the pulse formation. It was quite surprising because on the trailing edge of the pulse there is no shaping action of the absorber. There is even net gain, because the loss caused by the absorber is very small for the trailing edge, assuming a fully saturated absorber. Thus one might expect that this net gain would either prevent the pulse from getting so short or destabilize it by amplifying its trailing wing more and more.

In the femtosecond regime I considered soliton formation to be responsible for stable pulse generation [77] but others expected some additional faster mechanism inside the SESAM. The problem was finally resolved once I moved to ETH Zurich, where my group performed additional experiments with an actively modelocked Nd:glass laser using similar laser conditions as before with SESAM modelocking. Again much shorter pulses were obtained than predicted before and we could explain that result with soliton modelocking $[78,79]$ which was then extended to SESAM modelocking [74-76].

In soliton modelocking soliton-like pulse shaping leads to stable pulsing even in the presence of a considerable open net gain window before and after the pulse. The pulse is not any longer shaped dominantly by the saturable absorber or the active loss modulation, but they are still essential for pulse stability. Note that this regime of operation is significantly different from what had been previously discussed 
before in the context of the dye lasers (Fig. 1a), where the interplay between loss and gain saturation always leads to a short net gain window in time. In addition, previous numerical simulations showed that soliton formation can only lead to pulses of about a factor of two shorter than without soliton-like pulse shaping before the laser becomes unstable [80]. The same was predicted to be true for the fast saturable absorber (Fig. 1b) [72]. The open net gain windows that form in these cases are only about one to two pulse width long. For soliton modelocking, instead, the net gain window can remain open for 10 to 30 times the pulse duration depending on the specific laser parameters. Therefore, soliton-modelocked Ti:sapphire lasers stabilized with slow SESAMs then were used for the generation of pulses as short as 13 fs-no KLM with its critical cavity alignment was necessary for this result [76]. Soliton modelocking substantially relaxed the requirements for the SESAM parameters in the femtosecond regime where typically sufficient SPM can be generated for stable soliton formation. Generally these pulses are ideal solitons with excellent stability both in the time and frequency domain.

In the picosecond regime, experiments with SESAMmodelocked solid-state lasers also showed that even without soliton effects, the pulse duration can be at least 20 times shorter than the absorber recovery time. To understand why stable pulses with a duration far below the absorber recovery time are possible, we had to consider that the action of the absorber steadily delays the pulse [73]: it attenuates mostly its leading wing, thus shifting the pulse center backwards in each cavity round-trip. This shift does not occur to trailing edge of the pulse, because then the absorber is fully saturated already. Thus the pulse is constantly moving backward and can swallow any noise growing behind itself, which means that this noise has only a limited time in which it can experience gain before it merges with the pulse itself. The same mechanism can also prevent the trailing edge of the pulse from growing and ultimately stable pulses are obtained.

\section{Kerr-lens modelocking (KLM)}

KLM, discovered by the Sibbett group [3], was a key breakthrough and allowed the community to push the frontier of ultrashort pulses to the few femtosecond regime without any external pulse compression. Because the Kerr lens produces a "nonresonant" saturable absorber, it is inherently broadband, broader than any other saturable absorber available today. Thus compared to the ultrafast dye lasers, for which only pulses as short as $27 \mathrm{fs}$ with around $10 \mathrm{~mW}$ average power were generated [81], pulses around 5 to $6 \mathrm{fs}$ with hundreds of milliwatts of average power can be produced with Ti:sapphire lasers [10,13]. In addition, very broad tunability with sub-100-fs pulses became possible for the first time.
For time-resolved spectroscopy, these features had a major impact, since this laser provided more flexibility for different material parameters.

However, despite these advantages, KLM has some significant drawbacks. Generally, KLM lasers are not selfstarting. Restrictive cavity designs [82, 83] allow for better starting conditions, but this generally requires submillimeter precision on the cavity mirror alignments, a very clean environment to minimize intracavity losses, and a laser cavity operated close to the stability limit. Additionally, optimization for improved starting conditions conflict with the goal of achieving the shortest possible pulses, since the Kerr effect is too strong for very short pulses when the cavity is optimized to react sensitively to long pulses during the startup phase. In the picosecond regime, KLM lasers tend to be less stable because the Kerr lens becomes too weak. Today KLM is normally used for Ti:sapphire laser oscillators in the sub-10-femtosecond regime and for ultrabroad tuning. Typically a small mechanical shaker is mounted at one of the cavity mirrors to start the modelocking process, which is well sustained once started as long as the cavity parameters are adjusted correctly. Otherwise SESAMs have taken a more important role in ultrafast solid-state lasers.

KLM was an accidental discovery as discussed in Sect. 2.3. The postdeadline paper by Sibbett's group at CLEO in 1990 [68] had a major impact and "confidential" copies of their paper were showing up on fax machines even a few days before the conference. The presentation room at CLEO was fully packed with people pushing through the door. Afterwards many of us in the community went back to our labs and tried to reproduce his result, which at that time was called "magic" modelocking. I was in a good starting position having a working RPM Ti:sapphire laser (see Sect. 2.2) which was different from Sibbett's laser. Generally I had problems with the transverse mode stability of the Ti:sapphire laser and therefore had introduced an adjustable intracavity aperture inside the main cavity. One evening, by accident, I discovered that I could change the pulse duration from picosecond to femtosecond by simply partially closing this aperture. This could be reproduced as many times as I wanted and ultimately gave me the clue for the explanation of "magic" modelocking: the modelocking mechanism was based on the self-focusing effect inside the Ti:sapphire rod (see Ref. [61] explanation in second last paragraph) and no higher order spatial modes were required. This was later referred to as KLM (a name given by Coherent Inc.) [84]. With RPM I had a reliable starting mechanism for KLM and the aperture enabled a fast saturable loss modulation because the intensity dependent Kerr lens inside the Ti:sapphire rode reduced the cavity mode size at my aperture, thus introducing less loss for higher intensities (i.e. a stronger Kerr lens) than for lower intensities (i.e. no significant Kerr lens). I did not have to misalign the laser cavity such that the increased 
noise would ultimately start KLM because RPM provided a reliable starting mechanism. This paper also motivated Piché to formulate a more detailed analysis [85]. Many more papers followed with different starting mechanisms and optimized cavity designs that improved the limited self-starting behavior of KLM Ti:sapphire lasers.

\section{Semiconductor saturable absorber mirror (SESAM)}

SESAMs have made key contributions to the performance of ultrafast solid-state lasers and their improvements. The SESAM devices are a family of optical devices that allow for very simple, self-starting passive modelocking of ultrafast solid-state lasers. The SESAM design is a multi-disciplinary effort involving key understanding of both semiconductor and solid-state laser physics, and has resulted in new unprecedented performance improvements in terms of pulse widths, average power, and repetition rates in these lasers. These performance improvements and the implementation of the SESAM device also has allowed for many new practical application and commercialization of these lasers, increasing their use not only in R\&D environments but also in medical, industrial, metrology and communications applications.

More precisely, the SESAM device is based on the nanotechnology of semiconductor epitaxial material deposition, allowing control of layers of material down to the subnanometer accuracy. This allows for very precise optical devices with practically complete control of the key design parameters-magnitude and phase of the optical absorption and reflection, plus adjustment of key parameters such as the saturation fluence (energy level where the device saturates) and its temporal response (how fast the absorber recovers). The optical characteristics of the modulation depth, saturation fluence, and nonsaturable loss are key for proper laser performance. They are used as a direct input into theoretical guidelines to guarantee suppression of unwanted Q-switching instabilities with relatively simple expressions relating controllable design features of the laser and the SESAM device such that Q-switched modelocking can be avoided $[52,53]$. Once the device design is optimized $[86,87]$, a semiconductor wafer fabrication allows these devices to be produced economically, and ultimately in large volumes.

\subsection{Innovation and evolution of the SESAM concept}

The evolution of the concept of the SESAM device started with RPM (see Sect. 2). To understand and appreciate this evolution it helps to be aware of the stand of knowledge 20 years ago (see Sect. 2). For RPM, I used an early SESAM design in a coupled cavity, however, this SESAM had a far too large modulation depth and loss, due to its 75 quantum wells, to be introduced inside the main cavity - and in any case we were all somewhat stuck in the assumption that a simple intracavity saturable absorber would not work for a solid-state laser with a small gain cross section. Nevertheless the more detailed understanding of how RPM worked helped me to design the first working intracavity saturable absorber device, which I called the antiresonant Fabry-Pérot saturable absorber (A-FPSA) - in retrospect not a good choice for an acronym (see Sect. 4.2).

Figure 2 shows the evolution from RPM to the innovation of the first SESAM device. The RPM operation can be understood based on the intensity dependent coupledcavity reflectivity $R_{\mathrm{nl}}$ [88] (Fig. 2b). From inside the main cavity this coupled cavity looks like a Fabry-Pérot reflectivity, which shows a different intensity dependence for an amplitude (Fig. 2c) and a phase (Fig. 2d) nonlinearity. For an amplitude nonlinearity (Fig. 2c) — the case for RPM-the laser experiences an intensity dependent reflectivity change at the maximum reflectivity, which corresponds to the antiresonance of the Fabry-Pérot. In contrast to a phase nonlinearity (Fig. 2d) - the case for APM — an intensity dependent
Fig. 2 Operation principle of resonant passive modelocking (RPM): (a) schematic of a cw laser cavity with a laser gain element and the linear cavity. (b) Schematic of the coupled-cavity design with an saturable absorber inside. (c), (d) Intensity dependent nonlinear reflectivity $R_{\mathrm{nl}}$ of the coupled cavity as seen from inside the main cavity for an amplitude nonlinearity (c) and a phase nonlinearity (d) inside the coupled cavity. Explanation is according to Ref. [88]

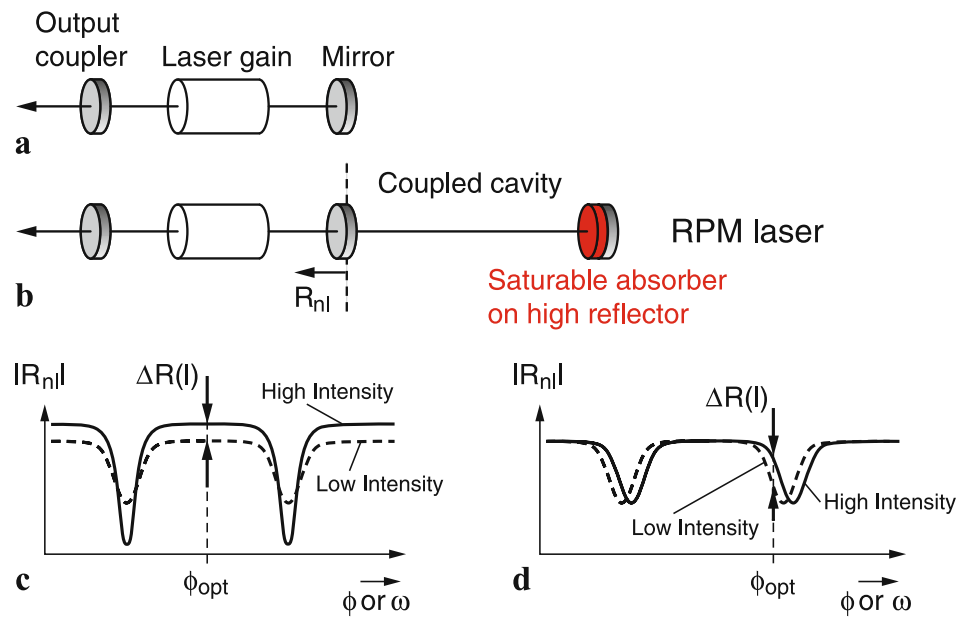


reflectivity is only obtained detuned away from maximum reflectivity, which requires an external cavity length control and interferometric stability. This simply explains why RPM in contrast to APM does not need any active cavity length stabilization for stable modelocking. The coupled cavity in RPM therefore can be considered like an intracavity antiresonant Fabry-Pérot saturable absorber (A-FPSA) as shown schematically in Fig. 3 [1]. Based on the RPM Nd:YLF laser parameters [88] for which I had $R_{\text {top }}=98 \%$ (Fig. 3a) and $50 \mathrm{InGaAs} / \mathrm{GaAs}$ quantum wells grown at low temperatures with molecular beam epitaxy (MBE) (red in Fig. 3a) on top of a high-reflecting GaAs/AlAs dielectric Bragg reflector (i.e. $R_{\text {bottom }}=100 \%$ in Fig. 3 ), I then designed the first A-FPSA device according to Fig. 3b, which worked and resulted in stable self-starting passive modelocking of a Nd:YLF laser generating 3.3 ps pulses [1].

It is maybe amusing to note that the Q-switching modelocking (QML) threshold (i.e. when the laser becomes truly cw modelocked without any Q-switching) was rather high with this first SESAM device, such that I even cracked part of the Nd:YLF crystal due to the high pump power needed to overcome this limit. Another interesting note is that I used low-temperature MBE growth, since the large numbers of InGaAs quantum wells, which are not lattice matched on GaAs, started to degrade the surface quality at normal MBE

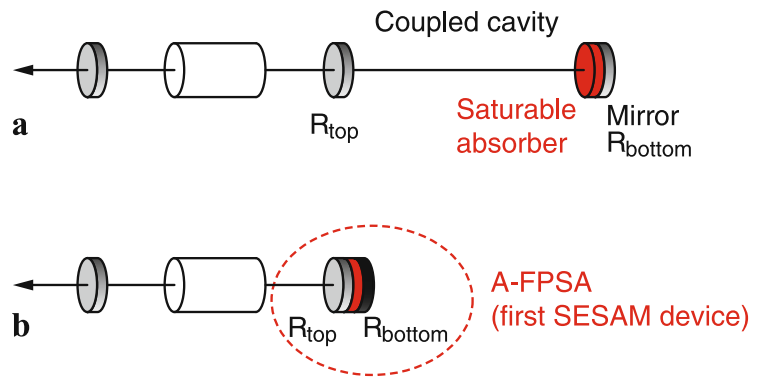

Fig. 3 Invention of the first SESAM device the antiresonant Fabry-Pérot saturable absorber (A-FPSA) published in 1992 [1]. (a) Schematic RPM cavity with coupled cavity. (b) Schematic linear cavity with intracavity saturable absorber, which can be considered as an A-FPSA in analogy to the nonlinear reflectivity $R_{\mathrm{nl}}$ of the coupled cavity (Fig. 2) growth temperature and introduced too much nonsaturable scattering losses inside the laser cavity. Only later did I realize that this had the additional benefit of reducing the recovery time from the nanosecond regime (for defect-free materials) to around $10 \mathrm{ps}$, which was necessary for the few picosecond pulse generation.

With this first SESAM design, I then had a device that allowed me to change many key parameters as I wished, benefiting from semiconductor material properties and growth techniques. The different A-FPSA structure design parameters and their variations have been discussed in Refs. [92] and [86]. Ultimately the top reflector of the A-FPSA (Fig. 3b) could be scaled to $0 \%$ replacing $R_{\text {top }}$ with an AR coating (Fig. 4) which reduced the number of quantum-well saturable absorbers to only one which was embedded inside the lower high-reflecting Bragg reflector (Fig. 4) [89]. In comparison to all early attempts to passively modelock solid-state lasers, the modulation depth was only a percentage or even sub-percentage level, and therefore the thick 50-quantum-well absorber embedded inside the high-finesse A-FPSA, without the high top reflector, would have generated far too large a modulation depth, driving the laser into Q-switching instabilities [52].

During these device parameter studies it became clear that in principle this A-FPSA device can be replaced by any nonlinear semiconductor mirror with the correct nonlinear amplitude and phase reflectivity response to prevent Qswitching instabilities. As the operating principles have become better understood, the device was ultimately monolithically integrated as a reflective end mirror into the laser cavity, and I referred to it as the SESAM in 1996 [2]. The functionality of the SESAM was extended to include a broader range of performance and wavelength ranges, such as dispersion compensation, ultra-broadband reflectivity, etc. For example, both negative GDD and nonlinear intensity reflectivity modulation was designed into a SESAM for the first time in 1996 [93]. We more recently reviewed the different design structures for SESAMs with low saturation fluence in Ref. [87]. Today we typically distinguish between high or low finesse designs (in analogy to the A-FPSA design principles where we either use a high or low top reflector $\left.R_{\text {top }}\right)$ and between antiresonant or resonant designs
Fig. 4 Scaling of the A-FPSA to a single quantum-well absorber in $1995[89,90]$

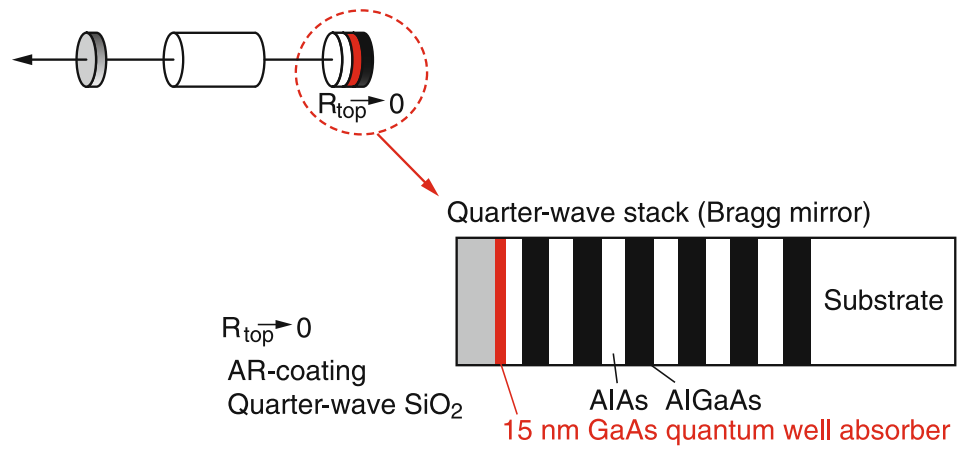




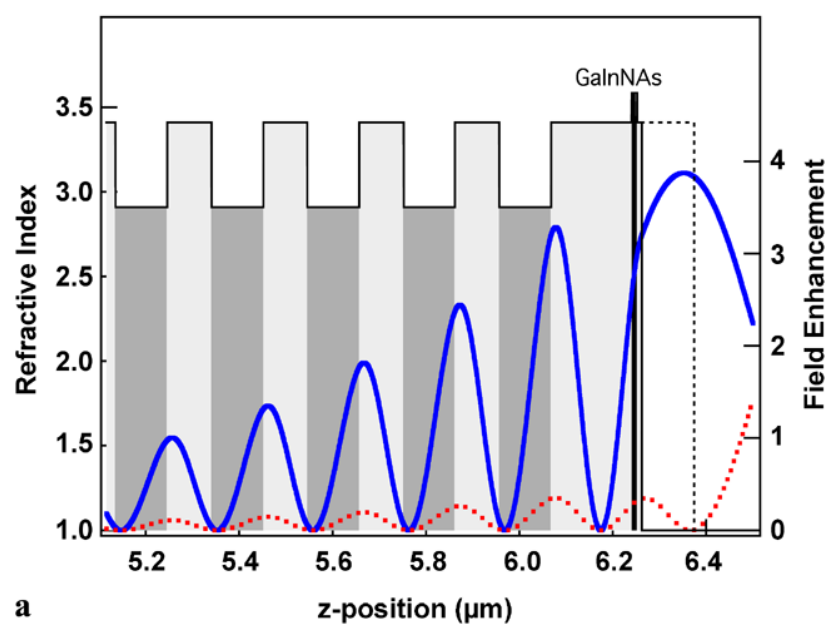

Fig. 5 Antiresonant versus resonant SESAM design: Example is given for a GaInNAs quantum-well SESAM [91] for which the top part of the multi-layer structure is shown with the refractive index profile (a). The dashed line indicates the increased thickness of the last layer which changes the SESAM design from a resonant to an antiresonant design. In blue and red we show the intensity distribution within the top part of the device for the resonant (blue) and the antiresonant (red)

as shown in Fig. 5. But in principle any values in between these extremes can be used and have different trade-offs as discussed in more details in Ref. [87].

\subsection{Choosing the correct acronyms}

The common accepted guidelines for "creating a new acronym" in science is that something new has to be discovered or invented to justify a new name. In my case the SESAM was a new intracavity saturable absorber device that solved the long-standing Q-switching problem for solidstate lasers. Therefore, I found it appropriate to create such an acronym and today this has also been widely accepted in the scientific community. The SESAM is in my opinion a relatively good and a very appropriate acronym, which describes the most important characteristics of this intracavity saturable absorber device that are based on semiconductor nonlinearities embedded inside a high-reflecting mirror. Additionally it is easy to pronounce. The SESAM is a multi-layer structure optimized for a certain amplitude and phase reflectivity response and therefore has a large number of different specific layer structures as a possible solution. The semiconductor material has been so successful because the absorber cross section is around $10^{-14} \mathrm{~cm}^{2}$ whereas the diode-pumped solid-state laser gain cross section is typically around $10^{-19}$ to $10^{-22} \mathrm{~cm}^{2}$. This leads to a significantly lower saturation energy of the absorber, which is greatly beneficial for stable modelocking of such lasers [52]. Thus the saturation fluence of a SESAM can be designed to be in the range of $1 \mu \mathrm{J} / \mathrm{cm}^{2}$ without pushing it too much into resonance [87].

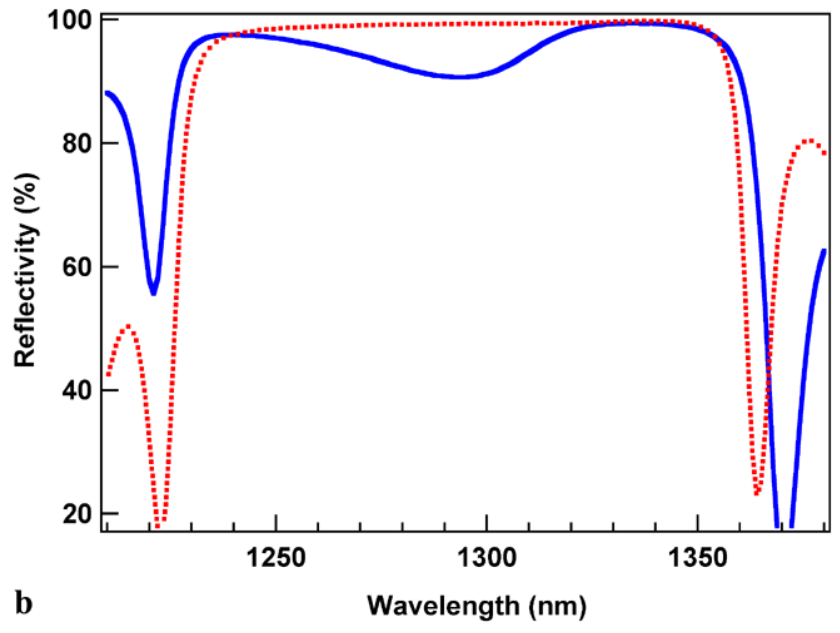

design, which clearly shows about a 10 times increased intensity inside the quantum-well absorber in the resonant design. (b) Measured linear reflectivity for the two SESAM designs where for the resonant design (blue) a clear reflectivity dip appears at resonance because of the increased loss of the quantum-well absorber with higher field enhancement

When I started this work, I first introduced a rather poor acronym (with the benefit of hindsight): the A-FPSA. First of all it was hardly pronounceable, and also defined a much too restrictive device design (see Sect. 4.1). For about the first three years after its introduction, I was the only one using such devices to passively modelock diode-pumped solidstate lasers, since generally the different research communities did not mix and there were either experts in "semiconductor nonlinearities" or "diode-pumped solid state lasers", but very seldom experts in both. At the beginning I was very often told that this was a rather complicated approach, and many researchers assumed that the A-FPSA would have some serious damage issues (which we now know is not an issue when the device is not driven too far above the saturation fluence). Optimized SESAMs did not show damage even at a fluence of $0.2 \mathrm{~J} / \mathrm{cm}^{2}$ and an peak intensity of $>400 \mathrm{GW} / \mathrm{cm}^{2}$ [94]. Motivated by my many successful results applying this technique to different lasers, more groups started to get involved in 1995. Some of them created new acronyms such as saturable Bragg reflector (SBR) [95] or even much later saturable absorber mirror (SAM).

As a general observation, I have now observed more than a few cases where there is an attempt to try to push forward a result as a new idea or new invention even when it may not be justified, be coming up with a new acronym. Unfortunately such actions sometimes become successful and may dilute the real information behind how a breakthrough started. This is even more of an issue when no acronym or a bad acronym is given to the original invention. This has happened many times in the history of science, and just within the present context of ultrafast solid-state lasers the "KLM" 
and "APM" acronyms are other examples. Ultimately it is also the ethical obligation of our research community to honor and cite the original inventors and their original papers.

\section{Frequency comb stabilization}

Ultrafast solid-state lasers enabled an important breakthrough in frequency metrology resulting in a Nobel prize in 2005 for J.L. Hall and T.W. Haensch "for their contribution to the development of laser-based precision spectroscopy, including the optical frequency comb technique" [96]. Here again two different research communities that traditionally did not interact much, solved an important problem in their specific fields with a cross-disciplinary approach.

For frequency metrology the stable frequency comb generated by femtosecond modelocked solid-state lasers provided one simple phase coherent link between optical frequencies and microwave frequencies, which can easily be counted with very high precision [97-99]. This enables many different applications [100-102] one of which is a more accurate clock because time is defined by the number of cycles of a perfectly periodic signal within one specific time interval. So the shorter the cycle period or the higher the frequency the more accurate this time interval can be defined. Moving from a microwave frequency of around $10 \mathrm{GHz}$ to optical frequencies in the $100 \mathrm{THz}$ regime improves this accuracy by 4 orders of magnitude and therefore the more accurate optical clocks are expected to address many new applications from gravity meters to testing of the physical constants over time.

For ultrafast lasers we have reached a pulse duration close to the fundamental limit of one optical cycle [103] (see also Sect. 1.1). Already during a Gordon conference on Nonlinear Optics and Lasers in 1995, many of the leading ultrafast laser experts discussed the upcoming challenge to stabilize the electric field underneath the pulse envelope, which only contains one to two optical cycles (Fig. 6). We did not come up with a solution at that time but we were aware that a

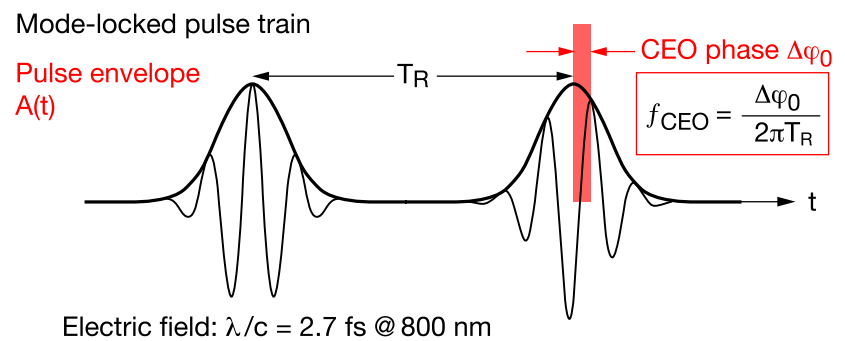

$$
E(t)=A(t) \exp \left(i \omega_{c} t+i \varphi_{0}(t)\right)
$$

Fig. 6 Time domain: pulse envelope $A(t)$ and electric field $E(t)$ with the carrier-envelope offset (CEO) phase $\Delta \varphi_{0}$ and CEO frequency $f_{\text {CEO }}$. How to stabilize the electric field underneath the pulse envelope was considered the "holy grail" problem for ultrafast lasers modelocked laser only stabilized the pulse envelope (which e.g. was well documented with Haus's master equations for modelocking theories). We expected that the electric field underneath the pulse envelope must exhibit strong fluctuations, but at that point it was not clear how we could characterize and stabilize them. We even considered the solution to this problem as the "holy grail" for ultrafast lasers. The holy grail turned out to be the stabilization of a frequency comb as well $[25,99,104]$.

Let me explain in more detail where the ultrafast community came from with regards to this "holy grail". In the 1980s, Dave Bloom at Stanford made pioneering contributions to the synchronization of actively modelocked picosecond lasers to a microwave frequency reference [105, 106], which he then applied to electro-optic sampling of GaAs integrated circuits [45]. I did my Ph.D. work in his group and explored to extend this synchronization technique to femtosecond fiber Raman soliton lasers [107, 108] to improve the time resolution in electro-optic sampling, which turned out not to work due to the poor noise properties of these lasers [109]. It was very well understood at that time, that modelocked lasers produce an equidistant pulse train in the time domain and the equivalent frequency comb in the frequency domain (Fig. 7a) which is simply based on the Fourier analysis which transforms a delta comb (or Dirac comb) in the time domain again into a delta comb in the frequency domain [110]

$$
\begin{aligned}
& E_{\text {train }}(t)=E(t) * \sum_{m=-\infty}^{\infty} \delta\left(t-m T_{R}\right) \Leftrightarrow \\
& \tilde{E}_{\text {train }}(f)=\tilde{E}(f) \sum_{m=-\infty}^{\infty} \delta\left(f-m f_{\text {rep }}\right)
\end{aligned}
$$

where $*$ describes the convolution, $E_{\text {train }}(t)$ is the electric field of the pulse train, $E(t)$ of a single pulse in the time domain and $\tilde{E}_{\text {train }}(f)$ resp. $\tilde{E}(f)$ the corresponding fields in the frequency domain, $\delta(t)$ the delta function (or more precisely the Dirac delta distribution), $T_{R}$ the pulse repetition period, $f_{\text {rep }}$ the pulse repetition frequency. Intensity and timing jitter noise can then easily be included (see e.g. Eq. 1 in Ref. [109]):

$$
I_{\text {train }}(t)=P T_{R}[1+N(t)] \sum_{m=-\infty}^{\infty} \delta\left(t-m T_{R}-J(t)\right)
$$

where we neglected the specific pulse form of the ultrashort pulses because we were only interested in the intensity and timing jitter noise, as measured with a fast photodetector and a microwave spectrum analyzer. $I_{\text {train }}(t)$ is the pulse intensity of the pulse train in the time domain, $P$ is the average power, $N(t)$ describes the normalized laser power fluctuations, and $J(t)$ the timing jitter which results from fluctuations of the pulse arrival time. Comparing Eqs. 1 and 2 
clearly demonstrates that any timing jitter directly couples into fluctuations of the spacing within the optical frequency comb (Eq. 1) (i.e. corresponds to a "breathing" of the comb lines). Therefore it is absolutely necessary to stabilize the timing jitter to obtain a stable optical frequency comb spacing. But this is not sufficient for a fully stabilized frequency comb that also has an additional degree of freedom with the translation of the full frequency comb (Fig. 7a).

Immediately after my invited talk at the "Jahrestagung der Deutschen Gesellschaft für angewandte Optik" in 1998, I was approached by Harald Telle for a collaboration on frequency comb stabilization. Telle comes from the metrology community and my group at that time had the world-record pulse duration from passively modelocked Ti:sapphire lasers. We then characterized the bandwidth of our Ti:sapphire laser, which however did not have sufficiently broad bandwidth to support the $f$-to- $2 f$ interferometer technique (i.e. direct SHG/DFG in Ref. [25]) (Fig. 7b) to stabilize the frequency comb (see Fig. 4 in Ref. [25]). We then explored many different techniques, which required less optical bandwidth and published these studies in 1999 [25] (with some delays as the graduate student A.E. Dunlop had to stop her Ph.D. for health reasons). Our pioneering contribution to this field was also recognized by the Nobel prize 2005 committee (see Ref. [37] in the advanced information summary in Ref. [111]).

Using the delta comb formalism for modelocked lasers (Eqs. 1 and 2) shows in a straightforward manner how a fully stabilized frequency comb solves the "holy grail" problem. The frequency comb of a modelocked laser has two degrees of freedom: the "comb breathing" described with $f_{\text {rep }}$ and the "comb translation" described with $f_{\text {CEO }}$ as shown in Fig. 7a (see Eq. 11 in Ref. [112]):

$\tilde{E}_{\text {train }}(f)=\tilde{A}\left(f-f_{c}\right) \sum_{m=-\infty}^{\infty} \delta\left(f-m f_{\text {rep }}-f_{\text {CEO }}\right)$

where $f_{c}$ is the center or carrier frequency of the optical spectrum and $A$ is the pulse envelope given by

$$
E(t)=A(t) \exp \left(i \omega_{c} t+i \varphi_{0}(t)\right)
$$

where $\omega_{c}=2 \pi f_{c}$ and phase fluctuations $\varphi_{0}(t)$ result in different positions of the electric field maximum underneath the pulse envelope. The time domain of the pulse train of Eq. 3 then follows directly from the shift theorem of the Fourier transformation: for any given function $f(t)$ with its Fourier transform $\tilde{f}(\omega) \equiv F\{f(t)\}$ results $F\left\{e^{i \omega_{0} t} f(t)\right\}=$ $\tilde{f}\left(\omega-\omega_{0}\right)$ (see Eq. 10 in Ref. [112]):

$$
\begin{aligned}
& E_{\text {train }}(t) \\
& \quad=A(t) \exp \left(2 \pi i f_{c} t-2 \pi i f_{\mathrm{CEO}} t\right) * \sum_{m=-\infty}^{\infty} \delta\left(t-m T_{R}\right) .
\end{aligned}
$$
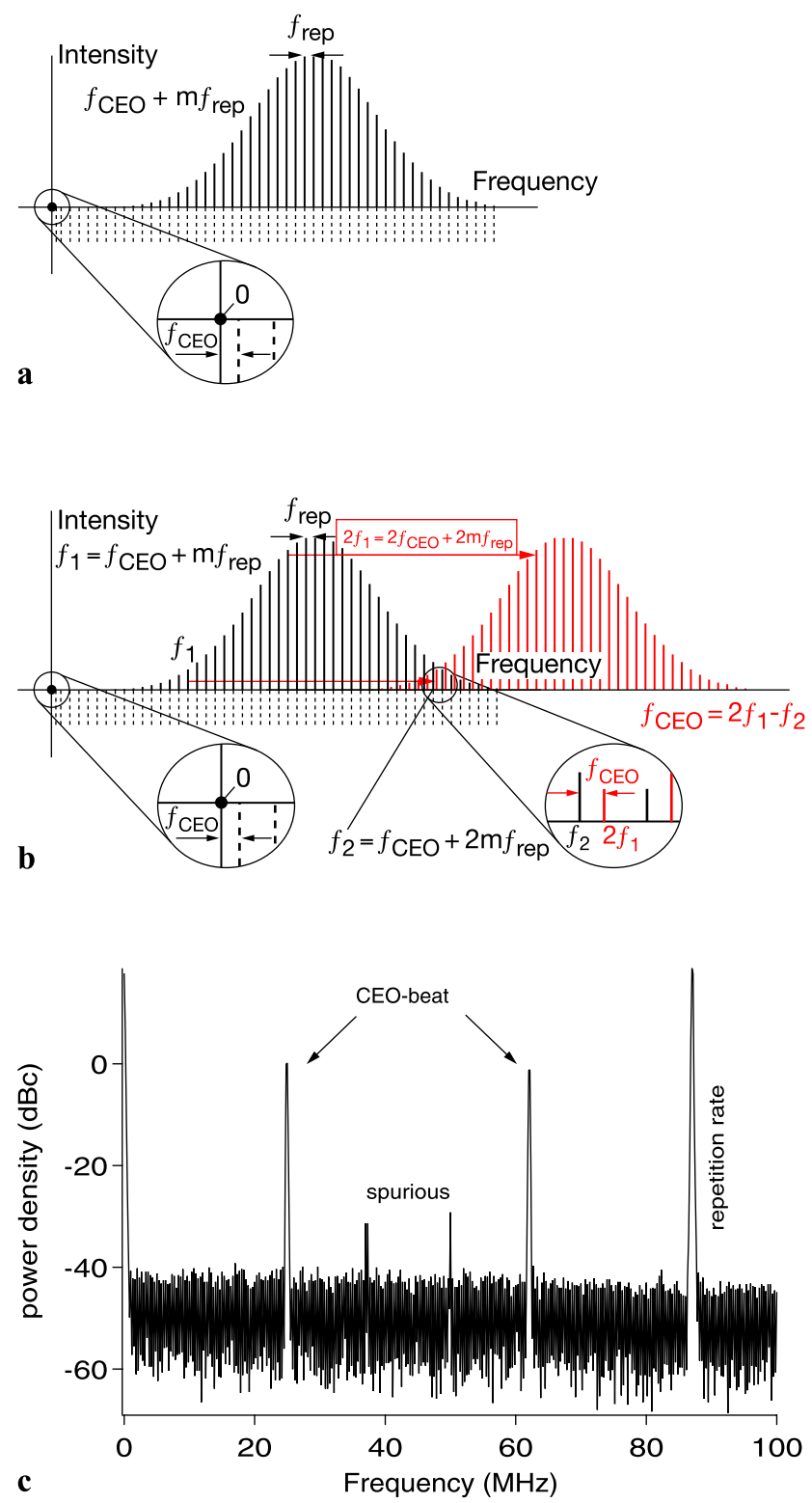

Fig. 7 Frequency domain: (a) frequency comb of a modelocked pulse train ( $f_{\text {rep }}$ is the pulse repetition frequency and $m$ an integer number). (b) Principle of $f$-to- $2 f$ interferometer technique (i.e. direct SHG/DFG in Ref. [25]) to stabilize the frequency comb. The second harmonic generated (SHG) frequency comb is overlapping with the fundamental for an octave-spanning spectrum. The frequency beat $2 f_{1}-f_{2}$ (i.e. difference frequency generation, DFG) between the fundamental $f_{1}$ and the SHG $f_{2}$ results in the carrier-envelope frequency beat signal: $2 f_{1}-f_{2}=\left(2 f_{\mathrm{CEO}}+2 m f_{\text {rep }}\right)-\left(f_{\mathrm{CEO}}+2 m f_{\text {rep }}\right)=f_{\mathrm{CEO}}$ [25]. (c) This CEO frequency signal is clearly detectable with a microwave spectrum analyzer [112]

Compared to Eq. 4 it follows that $f_{\mathrm{CEO}}$ determines the offset of the electric field (i.e. the "carrier") underneath the pulse envelope (Fig. 7a) and we therefore referred to this frequency as the carrier-envelope offset (CEO) frequency [25]. This CEO frequency can be measured with a $f$-to- $2 f$ in- 
terferometer technique [25] and a microwave spectrum analyzes (Fig. 7c).

\section{Novel applications enabled by ultrafast solid-state lasers}

Lasers generating short pulses-referred to as ultrafast lasers-enable many applications in science and technology. Numerous laboratory experiments have confirmed that ultrafast lasers can significantly increase the performance and even enable new applications in metrology, supercontinuum generation, and life sciences, which only work with ultrashort pulses. The elegance and simplicity of SESAMmodelocked lasers, particularly combined with new diodelaser-pumped schemes developed during the last twenty years, has resulted in new practical, commercially available ultrafast laser systems. These laser systems are being used extensively for applications in ultrafast laser systems, where expensive, power-hungry, maintenance-intensive lasers are being replaced. New commercial applications are emerging in medical and bio-medical applications (multi-photon imaging, tissue ablation, corrective eye surgery), material processing (precision micro- and nanocutting/processing of materials), telecommunications (pulse generating laser for next-generation soliton-like signal transmission and alloptical signal processing), optical clocking of multi-core microprocessors and frequency metrology.

There are also benefits to the economy and the ecology: these lasers use much less resources to be built, and substantially less power and less waste heat to operate. This ultimately reduces the lifetime energy expense of these types of lasers.

As the technology becomes smaller, less expensive, more robust, it allows for the exploitation of "ultrafast" phenomena to become more and more common, ultimately entering our everyday lives-for example using ultrafast lasers in high-speed optical communications for increasing communications bandwidth worldwide, optical clocking of the next generation of multi-core personal desktop computers, transportable optical clocks for precision metrology applications, and many medical and bio-chemical applications for which the current ultrafast technology is still considered to be too expensive. SESAM-modelocked diode-pumped solid-state, fiber and semiconductor lasers will continue to evolve and will help to expand such applications.

\section{Outlook}

In conclusion I can predict that we will see continued progress in ultrafast solid-state lasers both in their performance and cost reduction. There are many interesting applications that drive this development. In the near future our research efforts in my group are concentrating on two main activities: pulse energy scaling towards $100 \mu \mathrm{J}$ and compact gigahertz femtosecond pulse generation.

I expect that we will achieve femtosecond and picosecond pulses with more than $100 \mu \mathrm{J}$ directly out of a laser oscillator. A pulse energy of $100 \mu \mathrm{J}$ at $5 \mathrm{MHz}$ pulse repetition rate requires an average output power of $500 \mathrm{~W}$, which means that diode-pumped thin-disk lasers are ideally suited for this goal. Novel Yb-doped solid-state laser materials will hopefully enable sub-100-femtosecond pulse generation [113] which are particularly interesting for high laser field application and attosecond science [17, 114].

Compact diode-pumped solid-state lasers in the 5- to $20-\mathrm{GHz}$ regime need to generate sufficiently high peak power with femtosecond pulses to support broad frequency combs. First gigahertz femtosecond pulses have been generated using diode-pumped Yb:KYW [115] and Yb:KGW [116] lasers. Also very impressive results have been obtained with femtosecond VECSELs, although the average power is still limited in this regime [29]. Gigahertz SESAM modelocking requires a faster saturable absorber because soliton modelocking becomes less effective at these high pulse duty cycles and correspondingly lower peak powers. Faster SESAMs have successfully been produced at the low energy edge of the exciton resonance where the ac Stark effect contributes a fast nonlinearity to the SESAM response $[117,118]$. In addition, quantum-dot instead of quantumwell SESAMs seem to exhibit a faster response time [119], even though this apparently depends critically on the growth parameters [120]. Furthermore, nonlinearities in fibers can be further optimized with longer pulse duration, as demonstrated recently with a femtosecond Er:Yb:glass laser [26]. In addition, an improved concept for frequency comb stabilization has been reported recently as well [121]. All those efforts will ultimately make frequency comb applications much more affordable and practical.

Acknowledgements More than 40 graduate students and many postdocs have made essential contributions to my research efforts since I have joined the physics faculty at ETH Zurich, Switzerland in March 1993. I owe particular gratitude to my more senior postdocs who stayed for several years in my group before starting their own independent research efforts, such as Franz Kaertner, Guenter Steinmeyer, Ruediger Paschotta and my more recent senior postdocs Lukas Gallmann and Thomas Suedmeyer. I also benefited from many international collaboration, specifically with the group of Prof. Guenter Huber from University of Hamburg, Prof. David Hanna und Prof. Anne Tropper from University of Southhampton, Prof. Orazio Svelto and Prof. Sandro de Silvestri from the Politecnico di Milano. Also I benefited from substantial financial support from the Swiss National Science Foundation (SNSF), the Swiss Confederation's innovation promotion agency (CTI), different European research programs and from ETH with their support for a new clean-room facility (FIRST lab).

Open Access This article is distributed under the terms of the Creative Commons Attribution Noncommercial License which permits any noncommercial use, distribution, and reproduction in any medium, provided the original author(s) and source are credited. 


\section{References}

1. U. Keller, D.A.B. Miller, G.D. Boyd, T.H. Chiu, J.F. Ferguson, M.T. Asom, Opt. Lett. 17, 505 (1992)

2. U. Keller, K.J. Weingarten, F.X. Kärtner, D. Kopf, B. Braun, I.D. Jung, R. Fluck, C. Hönninger, N. Matuschek, J. Aus der Au, IEEE J. Sel. Top. Quantum Electron. 2, 435 (1996)

3. D.E. Spence, P.N. Kean, W. Sibbett, Opt. Lett. 16, 42 (1991)

4. P.F. Moulton, J. Opt. Soc. Am. B 3, 125 (1986)

5. U. Keller, in Landolt-Börnstein. Laser Physics and Applications. Subvolume B: Laser Systems. Part I, ed. by G. Herziger, H. Weber, R. Proprawe (Springer, Heidelberg, 2007), p. 33

6. U. Keller, Prog. Opt. 46, 1 (2004)

7. U. Keller, Nature 424, 831 (2003)

8. U. Keller, A.C. Tropper, Phys. Rep. 429, 67 (2006)

9. R.L. Fork, C.H.B. Cruz, P.C. Becker, C.V. Shank, Opt. Lett. 12, 483 (1987)

10. D.H. Sutter, G. Steinmeyer, L. Gallmann, N. Matuschek, F. Morier-Genoud, U. Keller, V. Scheuer, G. Angelow, T. Tschudi, Opt. Lett. 24, 631 (1999)

11. U. Morgner, F.X. Kärtner, S.H. Cho, Y. Chen, H.A. Haus, J.G. Fujimoto, E.P. Ippen, V. Scheuer, G. Angelow, T. Tschudi, Opt. Lett. 24, 411 (1999)

12. U. Morgner, F.X. Kärtner, S.H. Cho, Y. Chen, H.A. Haus, J.G. Fujimoto, E.P. Ippen, V. Scheuer, G. Angelow, T. Tschudi, Opt. Lett. 24, 920 (1999)

13. R. Ell, U. Morgner, F.X. Kärtner, J.G. Fujimoto, E.P. Ippen, V. Scheuer, G. Angelow, T. Tschudi, M.J. Lederer, A. Boiko, B. Luther-Davies, Opt. Lett. 26, 373 (2001)

14. F.X. Kärtner, N. Matuschek, T. Schibli, U. Keller, H.A. Haus, C. Heine, R. Morf, V. Scheuer, M. Tilsch, T. Tschudi, Opt. Lett. 22, 831 (1997)

15. R. Szipöcs, K. Ferencz, C. Spielmann, F. Krausz, Opt. Lett. 19, 201 (1994)

16. N. Matuschek, F.X. Kärtner, U. Keller, IEEE J. Sel. Top. Quantum Electron. 4, 197 (1998)

17. T. Südmeyer, S.V. Marchese, S. Hashimoto, C.R.E. Baer, G. Gingras, B. Witzel, U. Keller, Nat. Photonics 2, 599 (2008)

18. S.V. Marchese, C.R.E. Baer, A.G. Engqvist, S. Hashimoto, D.J.H.C. Maas, M. Golling, T. Südmeyer, U. Keller, Opt. Express 16, 6397 (2008)

19. J. Neuhaus, D. Bauer, J. Zhang, A. Killi, J. Kleinbauer, M. Kumkar, S. Weiler, M. Guina, D.H. Sutter, T. Dekorsy, Opt. Express 16, 20530 (2008)

20. C.R.E. Baer, C. Kränkel, C.J. Saraceno, O.H. Heckl, M. Golling, R. Peters, K. Petermann, T. Südmeyer, G. Huber, U. Keller, Opt. Lett. (2010 April 16 submitted)

21. L. Krainer, R. Paschotta, S. Lecomte, M. Moser, K.J. Weingarten, U. Keller, IEEE J. Quantum Electron. 38, 1331 (2002)

22. A.E.H. Oehler, T. Südmeyer, K.J. Weingarten, U. Keller, Opt. Express 16, 21930 (2008)

23. A. Oehler, M. Stumpf, S. Pekarek, T. Südmeyer, K. Weingarten, U. Keller, Appl. Phys. B 99, 53 (2010)

24. A. Schlatter, B. Rudin, S.C. Zeller, R. Paschotta, G.J. Spühler, L. Krainer, N. Haverkamp, H.R. Telle, U. Keller, Opt. Lett. 30, $1536(2005)$

25. H.R. Telle, G. Steinmeyer, A.E. Dunlop, J. Stenger, D.H. Sutter, U. Keller, Appl. Phys. B 69, 327 (1999)

26. M.C. Stumpf, S. Pekarek, A.E.H. Oehler, T. Südmeyer, J.M. Dudley, U. Keller, Appl. Phys. B (2009). doi:10.1007/ s00390-009-3854-8

27. S. Hoogland, S. Dhanjal, A.C. Tropper, S.J. Roberts, R. Häring, R. Paschotta, U. Keller, IEEE Photonics Technol. Lett. 12, 1135 (2000)

28. A. Aschwanden, D. Lorenser, H.J. Unold, R. Paschotta, E. Gini, U. Keller, Opt. Lett. 30, 272 (2005)
29. A.H. Quarterman, K.G. Wilcox, V. Apostolopoulos, Z. Mihoubi, S.P. Elsmere, I. Farrer, D.A. Ritchie, A. Tropper, Nat. Photonics 3, 729 (2009)

30. D.J.H.C. Maas, A.-R. Bellancourt, B. Rudin, M. Golling, H.J. Unold, T. Südmeyer, U. Keller, Appl. Phys. B 88, 493 (2007)

31. A.-R. Bellancourt, D.J.H.C. Maas, B. Rudin, M. Golling, T. Südmeyer, U. Keller, IET Optoelectron. 3, 61 (2009)

32. D.J.H.C. Maas, A.R. Bellancourt, M. Hoffmann, B. Rudin, Y. Barbarin, M. Golling, T. Südmeyer, U. Keller, Opt. Express 16, 18646 (2008)

33. A.-R. Bellancourt, Y. Barbarin, D.J.H.C. Maas, M. Shafiei, M. Hoffmann, M. Golling, T. Südmeyer, U. Keller, Opt. Express 17, 9704 (2009)

34. V.J. Wittwer, B. Rudin, D.J.H.C. Maas, H.D. Hoffmann, O.D. Sieber, Y. Barbarin, M. Golling, T. Südmeyer, U. Keller, MIXSEL: an integrated passively modelocked external-cavity semiconductor laser with $6.4 \mathrm{~W}$ average power, in 4th EPSQEOD Europhoton Conference, Hamburg, August 29-September 3, 2010

35. P. Kreuter, B. Witzigmann, D.J.H.C. Maas, Y. Barbarin, T. Südmeyer, U. Keller, Appl. Phys. B 91, 257 (2008)

36. G.J. Spühler, R. Paschotta, R. Fluck, B. Braun, M. Moser, G. Zhang, E. Gini, U. Keller, J. Opt. Soc. Am. B 16, 376 (1999)

37. B. Braun, F.X. Kärtner, U. Keller, J.-P. Meyn, G. Huber, Opt. Lett. 21, 405 (1996)

38. G.J. Spühler, R. Paschotta, M.P. Kullberg, M. Graf, M. Moser, E. Mix, G. Huber, C. Harder, U. Keller, Appl. Phys. B 72, 285 (2001)

39. R. Häring, R. Paschotta, R. Fluck, E. Gini, H. Melchior, U. Keller, J. Opt. Soc. Am. B 18, 1805 (2001)

40. A.J. DeMaria, D.A. Stetser, H. Heynau, Appl. Phys. Lett. 8, 174 (1966)

41. H.A. Haus, IEEE J. Quantum Electron. 12, 169 (1976)

42. D.J. Kuizenga, A.E. Siegman, IEEE J. Quantum Electron. 6, 694 (1970)

43. D.J. Kuizenga, A.E. Siegman, IEEE J. Quantum Electron. 6, 709 (1970)

44. J.D. Kafka, B.H. Kolner, T.M. Baer, D.M. Bloom, Opt. Lett. 9, 505 (1984)

45. K.J. Weingarten, M.J.W. Rodwell, D.M. Bloom, IEEE J. Quantum Electron. 24, 198 (1988)

46. J.-C. Diels, in Dye lasers principles: with applications, ed. by F.D.A.L. Hillman (Academic Press, Boston, 1990), p. 41

47. C.V. Shank, in Ultrashort Laser Pulses and Applications, ed. by W. Kaiser (Springer, Heidelberg, 1988), Chap. 2

48. F.M. Mitschke, L.F. Mollenauer, Opt. Lett. 12, 407 (1987)

49. M.N. Islam, E.R. Sunderman, C.E. Soccolich, I. Bar-Joseph, N. Sauer, T.Y. Chang, B.I. Miller, IEEE J. Quantum Electron. 25, 2454 (1989)

50. U. Keller, K.D. Li, B.T. Khuri-Yakub, D.M. Bloom, K.J. Weingarten, D.C. Gerstenberger, Opt. Lett. 15, 45 (1990)

51. K.J. Weingarten, D.C. Shannon, R.W. Wallace, U. Keller, Opt. Lett. 15, 962 (1990)

52. C. Hönninger, R. Paschotta, F. Morier-Genoud, M. Moser, U. Keller, J. Opt. Soc. Am. B 16, 46 (1999)

53. R. Grange, M. Haiml, R. Paschotta, G.J. Spuhler, L. Krainer, M. Golling, O. Ostinelli, U. Keller, Appl. Phys. B 80, 151 (2005)

54. L.F. Mollenauer, R.H. Stolen, Opt. Lett. 9, 13 (1984)

55. P.N. Kean, X. Zhu, D.W. Crust, R.S. Grant, N. Landford, W. Sibbett, Opt. Lett. 14, 39 (1989)

56. E.P. Ippen, H.A. Haus, L.Y. Liu, J. Opt. Soc. Am. B 6, 1736 (1989)

57. K.J. Blow, D. Wood, J. Opt. Soc. Am. B 5, 629 (1988)

58. U. Keller, T.K. Woodward, D.L. Sivco, A.Y. Cho, Opt. Lett. 16, 390 (1991)

59. U. Keller, W.H. Knox, H. Roskos, Opt. Lett. 15, 1377 (1990) 
60. H.A. Haus, U. Keller, W.H. Knox, J. Opt. Soc. Am. B 8, 1252 (1991)

61. U. Keller, G.W. 'tHooft, W.H. Knox, J.E. Cunningham, Opt. Lett. 16, $1022(1991)$

62. G.H.C. New, Opt. Commun. 6, 188 (1972)

63. H.A. Haus, IEEE J. Quantum Electron. 11, 323 (1975)

64. H.A. Haus, IEEE J. Quantum Electron. 11, 736 (1975)

65. H.A. Haus, J. Appl. Phys. 46, 3049 (1975)

66. N. Sarukura, Y. Ishida, H. Nakano, Opt. Lett. 16, 153 (1991)

67. Y. Ishida, N. Sarukura, H. Nakano, in Ultrafast Phenomena (OSA, Washington, 1990), PD11

68. D.E. Spence, P.N. Kean, W. Sibbett, in Conference on Lasers and Electro-optics (CLEO) (OSA/IEEE LEOS, Washington, 1990), CPDP10

69. R.L. Fork, B.I. Greene, C.V. Shank, Appl. Phys. Lett. 38, 617 (1981)

70. G.H.C. New, IEEE J. Quantum Electron. 10, 115 (1974)

71. U. Keller, W.H. Knox, G.W. 'tHooft, IEEE J. Quantum Electron. 28, $2123(1992)$

72. H.A. Haus, J.G. Fujimoto, E.P. Ippen, IEEE J. Quantum Electron. 28, 2086 (1992)

73. R. Paschotta, U. Keller, Appl. Phys. B 73, 653 (2001)

74. F.X. Kärtner, U. Keller, Opt. Lett. 20, 16 (1995)

75. I.D. Jung, F.X. Kärtner, L.R. Brovelli, M. Kamp, U. Keller, Opt. Lett. 20, 1892 (1995)

76. F.X. Kärtner, I.D. Jung, U. Keller, IEEE J. Sel. Top. Quantum Electron. 2, 540 (1996)

77. U. Keller, T.H. Chiu, J.F. Ferguson, Opt. Lett. 18, 1077 (1993)

78. D. Kopf, F. Kärtner, K.J. Weingarten, U. Keller, Opt. Lett. 19, $2146(1994)$

79. F.X. Kärtner, D. Kopf, U. Keller, J. Opt. Soc. Am. B 12, 486 (1995)

80. O.E. Martinez, R.L. Fork, J.P. Gordon, Opt. Lett. 9, 156 (1984)

81. J.A. Valdmanis, R.L. Fork, IEEE J. Quantum Electron. 22, 112 (1986)

82. G. Cerullo, S. De Silvestri, V. Magni, L. Pallaro, Opt. Lett. 19, 807 (1994)

83. G. Cerullo, S. De Silvestri, V. Magni, Opt. Lett. 19, 1040 (1994)

84. D.K. Negus, L. Spinelli, N. Goldblatt, G. Feugnet, in Advanced Solid-State Lasers, ed. by G. Dubé, L. Chase (Optical Society of America, Washington, 1991), p. 120

85. F. Salin, J. Squier, M. Piché, Opt. Lett. 16, 1674 (1991)

86. L.R. Brovelli, U. Keller, T.H. Chiu, J. Opt. Soc. Am. B 12, 311 (1995)

87. G.J. Spühler, K.J. Weingarten, R. Grange, L. Krainer, M. Haiml, V. Liverini, M. Golling, S. Schon, U. Keller, Appl. Phys. B 81, 27 (2005)

88. U. Keller, T.H. Chiu, IEEE J. Quantum Electron. 28, 1710 (1992)

89. L.R. Brovelli, I.D. Jung, D. Kopf, M. Kamp, M. Moser, F.X. Kärtner, U. Keller, Electron. Lett. 31, 287 (1995)

90. I.D. Jung, L.R. Brovelli, M. Kamp, U. Keller, M. Moser, Opt. Lett. 20, 1559 (1995)

91. V. Liverini, S. Schön, R. Grange, M. Haiml, S.C. Zeller, U. Keller, Appl. Phys. Lett. 84, 4002 (2004)

92. U. Keller, Appl. Phys. B 58, 347 (1994)

93. D. Kopf, G. Zhang, R. Fluck, M. Moser, U. Keller, Opt. Lett. 21, 486 (1996)

94. C.J. Saraceno, C. Schriber, M. Mangold, M. Hoffmann, O.H. Heckl, C.R.E. Baer, M. Golling, T. Südmeyer, U. Keller, Lowloss, high-damage threshold SESAMs for high pulse energy oscillators, in Europhoton Conference 2010, Hamburg, Germany, 2010
95. S. Tsuda, W.H. Knox, Souza, E.A.d., Jan, W.Y., Cunningham, J.E., Opt. Lett. 20, 1406 (1995)

96. J.L. Hall, T.W. Hänsch, http://nobelprize.org/nobel_prizes/lists/ 2005.html (2005)

97. S.A. Diddams, D.J. Jones, J. Ye, S. Cundiff, J.L. Hall, J.K. Ranka, R.S. Windeler, R. Holzwarth, T. Udem, T.W. Hänsch, Phys. Rev. Lett. 84, 5102 (2000)

98. J. Stenger, T. Binnewies, G. Wilpers, F. Riehle, H.R. Telle, J.K. Ranka, R.S. Windeler, A.J. Stentz, Phys. Rev. A 63, 021802 (2001)

99. R. Holzwarth, T. Udem, T.W. Hänsch, J.C. Knight, W.J. Wadsworth, P.S.J. Russell, Phys. Rev. Lett. 85, 2264 (2000)

100. T. Udem, R. Holzwarth, T.W. Hänsch, Nature 416, 233 (2002)

101. J. Ye, S.T. Cundiff, Femtosecond Optical Frequency Comb: Principle, Operation, and Applications (Springer, New York, 2005), p. 10013

102. T.W. Hänsch, Rev. Mod. Phys. 78, 1297 (2006)

103. G. Steinmeyer, D.H. Sutter, L. Gallmann, N. Matuschek, U. Keller, Science 286, 1507 (1999)

104. D.J. Jones, S.A. Diddams, J.K. Ranka, A. Stentz, R.S. Windeler, J.L. Hall, S.T. Cundiff, Science 288, 635 (2000)

105. M.J.W. Rodwell, K.J. Weingarten, D.M. Bloom, T. Baer, B.H. Kolner, Opt. Lett. 11, 638 (1986)

106. M.J.W. Rodwell, D.M. Bloom, K.J. Weingarten, IEEE J. Quantum Electron. 25, 817 (1989)

107. A.S. Gouveia-Neto, A.S.L. Gomes, J.R. Taylor, IEEE J. Quantum Electron. 24, 332 (1988)

108. J.D. Kafka, T. Baer, Opt. Lett. 12, 181 (1987)

109. U. Keller, K.D. Li, M.J.W. Rodwell, D.M. Bloom, IEEE J. Quantum Electron. 25, 280 (1989)

110. A.E. Siegman, Lasers (University Science Books, Mill Valley, 1986)

111. J.L. Hall, T.W. Hänsch, http://nobelprize.org/nobel_prizes/ physics/laureates/2005/press.html (2005)

112. F.W. Helbing, G. Steinmeyer, U. Keller, IEEE J. Sel. Top. Quantum Electron. 9, 1030 (2003)

113. T. Südmeyer, C. Kränkel, C.R.E. Baer, O.H. Heckl, C.J. Saraceno, M. Golling, R. Peters, K. Petermann, G. Huber, U. Keller, Appl. Phys. B 97, 281 (2009)

114. U. Keller, IEEE Photonics J. 2, 225 (2010)

115. S. Yamazoe, M. Katou, T. Adachi, T. Kasamatsu, Opt. Lett. 35, $748(2010)$

116. S. Pekarek, C. Fiebig, M.C. Stumpf, A.E.H. Oehler, K. Paschke, G. Erbert, T. Südmeyer, U. Keller, in Conference on Lasers and Electro-Optics (CLEO), 2010, paper CThI1

117. S. Hoogland, A. Garnache, I. Sagnes, J.S. Roberts, A.C. Tropper, IEEE Photonics Technol. Lett. 17, 267 (2005)

118. K.G. Wilcox, Z. Mihoubi, G.J. Daniell, S. Elsmere, A. Quarterman, I. Farrer, D.A. Ritchie, A. Tropper, Opt. Lett. 33, 2797 (2008)

119. E.U. Rafailov, S.J. White, A.A. Lagatsky, A. Miller, W. Sibbett, D.A. Livshits, A.E. Zhukov, V.M. Ustinov, IEEE Photonics Technol. Lett. 16, 2439 (2004)

120. D.J.H.C. Maas, M. Hoffmann, A.R. Bellancourt, B. Rudin, Y. Barbarin, M. Golling, T. Südmeyer, U. Keller, in Advanced SolidState Photonics (ASSP), 2009

121. S. Koke, C. Grebing, H. Frei, A. Anderson, A. Assion, G. Steinmeyer, Nat. Photonics (2010). doi:10.1038/NPHOTON.2010.91 